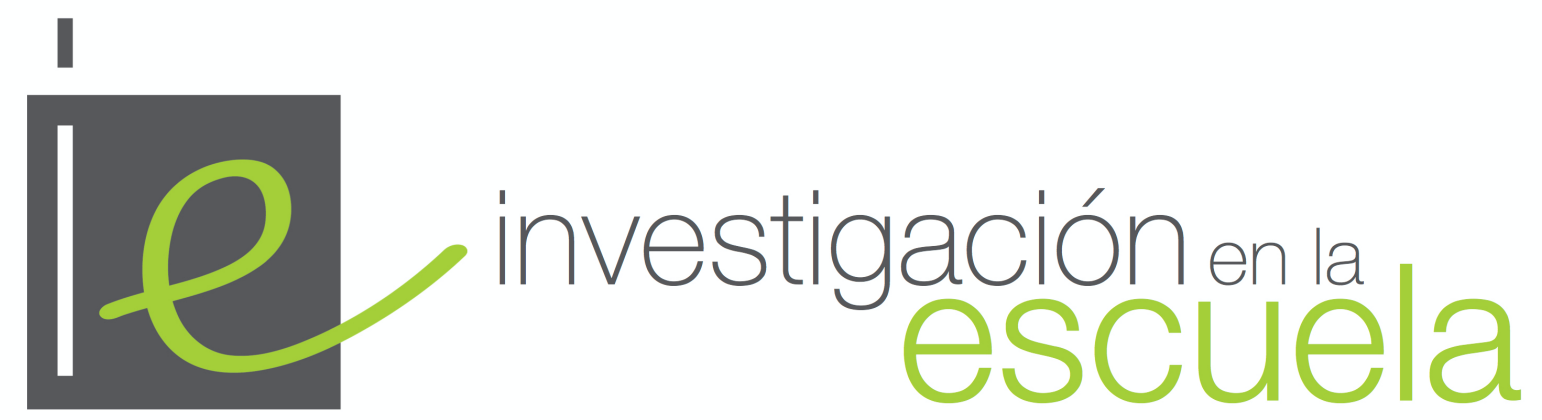

Revista internacional de investigación e innovación educativa

\title{
Educación patrimonial, intergeneracionalidad e interseccionalidad desde una perspectiva de género. Experiencia y conclusiones para la formación inicial de educadores y docentes
}

\author{
María del Consuelo Díez. Bedmar \\ Universidad de Jaén
}

España

Citación: Díez Bedmar, Ma . C. (2020). Educación patrimonial, intergeneracionalidad e interseccionalidad desde una perspectiva de género. Experiencia y conclusiones para la formación inicial de educadores y docentes. Investigación en la Escuela, 100, 55-70. DOI:

https://dx.doi.org/10.12795/IE.2020.1100.05

Resumen: El artículo presenta los resultados obtenidos en el desarrollo de dos propuestas intergeneracionales entre estudiantes de Grado y del Programa Universitario para Mayores de la Universidad de Jaén, en el contexto de asignaturas y temas patrimoniales, llevadas a cabo durante el curso académico 2018-2019. Aborda la importancia de la educación patrimonial desde la interseccionalidad y con perspectiva de género en el aprendizaje y desarrollo de constructos sociales. A partir del análisis de los discursos, tanto orales (con el alumnado mayor) como escritos, del alumnado de los distintos grupos que participaron en la experiencia, recogidos a partir de una ficha de registro de observación dirigida, se evidencia la importancia de estas propuestas para comprender que, en nuestra relación con el patrimonio, hay diferencias de apreciación y percepción (entre ellas por edad, sexo y roles de género asociados) y que debemos tener presente cómo nuestros 
estereotipos y prejuicios afectan al aprendizaje y a la construcción de una ciudadanía crítica, reflexiva y corresponsable con el patrimonio. Así mismo, los resultados muestran cómo, investigaciones de este tipo, nos proporcionan referentes básicos para los procesos de aprendizaje y enseñanza patrimoniales en Educación de Adultos, Educación Secundaria para Personas Adultas, Bachillerato para Adultos y los módulos de Formación Profesional que cada vez tienen más estudiantes mayores entre su alumnado.

Palabras clave: "Ciencias sociales"; "patrimonio intergeneracional”; "interseccionalidad"; "educación de adultos"; "interrelaciones"; "patrimonio cultural”; "investigación”.

\section{Heritage education, intergenerationality and intersectionality from a gender perspective.} Experience and conclusions for the initial training of educators and teachers

Abstract: The contribution presents the results obtained in the development of two heritage intergenerational proposals, carried out between undergraduate and University Program for Seniors students of the University of Jaén, in the context of subjects and heritage issues, during the 20182019 academic year. The importance of heritage education from intersectionality and with a gender perspective in the learning and development of social constructs is present. From the analysis of the speeches both oral (with older students) and written, of the students of the different groups that participated in the experience, collected from a directed analysis search form, the importance of that proposal has been evidenced in order to understand that there are differences of appreciation and perception (among them by age, sex and associated gender roles), stereotypes and prejudices in our relationship with heritage that affect the learning and the construction of a critical, reflective and coresponsible citizenship with heritage. Likewise, the results show how, investigations of this type provide us with basic references for the processes of heritage learning and teaching in Adult Education, Secondary Education for Adults, Baccalaureate for Adults and Higher level Vocational Training modules that increasingly have more older students among their students.

Key words: "Social science"; "heritage"; "intergenerational"; "intersectionality"; "adult education"; "interrelacions"; "cultural heritage"; "research".

\section{L'éducation au patrimoine, l'intergénérationnalité et l'intersectionnalité dans une} perspective de genre. Expérience et conclusions pour la formation initiale des éducateurs et des enseignants

Resumè: Cet article montre les données et les résultats obtenus dans l'élaboration de deux propositions intergénérationnelles, entre les étudiants de premier cycle et le Programme Universitaire pour les étudiants plus âgés de l'Université de Jaén, dans le contexte des sujets et des contenus patrimoniales, menées au cours de l'année scolaire 2018-2019. Il aborde l'importance de l'éducation au patrimoine sous la perspective de l'intersectionnalité et la perspective de genre dans l'apprentissage et le développement des concepts sociales. Sur la base de l'analyse des discours, tant oraux (avec les étudiants les plus âgés) qu'écrits, du corps étudiant des différents groupes qui ont participé à l'expérience, collectés à partir d'une fiche d'observation guidée. C'est évident la pertinence de ces propositions pour comprendre que, dans notre relation avec le patrimoine, il existe des différences d'appréciation et de perception (par âge, sexe et rôles de genre associés) et nous devons prendre en compte, comment nos stéréotypes et préjugés affectent l'apprentissage et la construction d'une citoyenneté critique, réfléchie et coresponsable avec le patrimoine. De même, les résultats montrent comment, enquêtes de ce type, nous fournissent des références fondamentales pour les processus d'apprentissage et d'enseignement du patrimoine dans l'éducation des adultes, l'enseignement secondaire pour adultes, le baccalauréat pour adultes et les modules de formation professionnelle qu'ils ont de plus en plus des élèves plus âgés parmi leurs étudiants. 
Mots-clés: "Sciences sociales"; "patrimoine"; "intergénérationnel”; "intersectionnalité"; "éducation des adultes"; "interrelations"; "patrimoine culturel"; "enquête".

\section{Introducción}

Desde distintos puntos de vista, dos hombres miran el mismo paisaje. Sin embargo, no en lo mismo. (Ortega y Gasset, 1923, p. 613)

La Agenda 2030 de la ONU para el Desarrollo Sostenible ${ }^{1}$ presenta la ciudadanía mundial, la diversidad cultural y el diálogo intercultural como principios globales del desarrollo sostenible, en particular en el objetivo 11 (ciudades patrimonio) y en el objetivo 4 (educación). Se marca entre sus objetivos, "acciones educativas en materia de patrimonio, promoviendo así la inclusión y la integración sociales" $(1$, a) y "sensibilizar al público acerca de la importancia del patrimonio cultural europeo a través de la educación y el aprendizaje permanente, atendiendo en particular a los niños, los jóvenes, las personas mayores, las comunidades locales y los grupos a los que resulta difícil llegar" (1, j). Estepa (2017, p. 48) recordaba que "Educar para la valoración y disfrute del patrimonio cultural y la defensa de su conservación y difusión" es una "finalidad específica de la enseñanza de la Historia, porque el trabajo sobre el patrimonio nos conecta con el pasado y facilita el desarrollo de métodos de análisis histórico".

Desde la educación patrimonial (Fontal, 2013; Fontal e Ibánez, 2015; Martín y Cuenca, 2015; Lucas, 2018) esta finalidad aparece como específica de la enseñanza de la Historia desde el análisis de los aprendizajes de las y los estudiantes, tanto en contextos formales como informales. En este sentido, Santisteban, González-Monfort \& Pagès (2019) proponen algunos objetivos fundamentales de una educación patrimonial crítica vinculados a la asociación con la identidad, la visibilización de colectivos, el desarrollo del pensamiento y la conciencia histórica.

La consecución de estos objetivos requiere una profunda reflexión crítica sobre nuestros propios posicionamientos y las categorías de pensamiento que empleamos y desarrollamos en la formación inicial del profesorado para que sea consciente de qué transmite y porqué y de qué mensajes procesan, cómo y porqué aquellas personas con quienes construyen conocimiento. Por eso, tener experiencias de aprendizaje en contextos diversos y en los que la intergeneracionalidad y la interseccionalidad con perspectiva de género estén presentes lleva a comprender las distintas maneras de relacionarse, identificarse e identificar elementos patrimoniales, así como los aprendizajes diversos que se producen.

\section{La necesaria intersección de categorías de análisis}

Sternberg (1986, citado en Martín, 1999) señalaba que el aprendizaje es más efectivo cuanto más significativo es para la persona, ya que "los adultos resuelven problemas y procesan la información en función del significado que éstos tienen para su desarrollo personal”. El patrimonio que nos rodea se erige como un tema significativo y controvertido que nos une a nuestro pasado, a nuestro presente y a la toma de decisiones hacia el futuro, que nos facilita reflexionar sobre los saberes escolares construidos que se nos transmitieron en las aulas, la construcción de nuestra identidad patrimonial y la influencia que tienen las categorías de pensamiento que priorizamos sobre nuestra percepción acerca del patrimonio y los valores patrimoniales.

\footnotetext{
${ }^{1} \mathrm{El}$ Informe mundial sobre la cultura para el desarrollo urbano sostenible (UNESCO, 2016) señala "Los responsables de la toma de decisiones deben basarse en la cultura para promover un desarrollo inclusivo, superando las deficiencias en los indicadores y en la medición del impacto, las limitaciones en la participación de los ciudadanos y la desigualdad de género".
} 
¿Qué narrativas aceptamos y elaboramos con respecto al patrimonio que nos rodea? Si bien desde la teoría queda clara la necesidad de analizar causas y consecuencias, desentrañar las causalidades históricas y realizar análisis críticos, para poner en valor e interiorizar los procesos y aprehenderlos es necesario planificar estrategias educativas, generar espacios de reflexión y encuentro de intereses diversos.

El diálogo intergeneracional (Bedmar 2003, 2010) permite el enriquecimiento mutuo de los aprendizajes, la ruptura de estereotipos y supone un espacio donde las diversidades son reconocidas como diferencias enriquecedoras, elementos fundamentales para la construcción de nuestra identidad. En este diálogo, la creación de las condiciones para una relación mayéutica presupone una escucha activa por parte de quienes intervienen y, en esa relación dialéctica, el diálogo con otros grupos de intereses diversos nos permite desarrollar nuestro espíritu crítico, reconocer la parcialidad de cada mirada al patrimonio y desarrollar nuestra competencia de aprender a aprender. Supone además establecer un enfoque intercultural en el que se produce una redefinición de la identidad patrimonial

La Teoría de la Interseccionalidad (Crenshaw, 1989) nos advertía de la incapacidad de ver en el sujeto un conjunto de identidades relacionadas entre ellas, lo que supone una visión parcial de la realidad. Ante el riesgo de reproducir las mismas relaciones de poder que han engendrado el estatus quo en jerarquías de saberes, conocimientos y personas (que la formación para una ciudadanía crítica debe plantearse), la educación patrimonial debería tener en cuenta múltiples categorías de análisis para el desarrollo de una identidad patrimonial interseccional que "(...) propicia que se hagan ostensibles las injusticias estructurales correlativas al capitalismo, el racismo y el sexismo. De esta manera, el enfoque interseccional va a dinamizar lo que se ha denominado como política de la pertenencia y va a cuestionar la heterodesignación" (Guerra, 2016, p. 207).

Por ello, la intersección de categorías de análisis debería tenerse en cuenta a la hora de realizar propuestas de educación patrimonial para la deconstrucción de las categorías normalizadoras y homogeneizantes de manera que, el futuro profesorado, sea consciente de su responsabilidad profesional y generacional: "History has to be rewritten in every generation because, although the past does not change, the present does; each generation asks new questions of the past, and finds new areas of sympathy as it re-lives different aspects of the experiences of its predecessors" (Hill, 1972, p. 13 citada en Díez Bedmar, 2019, p. 88).

\section{Método}

Para esta investigación partimos de la hipótesis de que las propuestas de educación patrimonial planificadas como encuentros intergeneracionales y donde estén presentes categorías e identidades interseccionales contribuyen a romper estereotipos, prejuicios y mitos vinculados a grupos de edad, nivel educativo y sexo-género.

Para contrastar la hipótesis, durante el curso académico 2018-2019 se planificaron espacios de encuentro intergeneracionales (Martins, Midão, Martínez, Dequech, Busse, Bertram, McDonald, Gilliland, Orte, Vives \& Costa, 2019), uno por cuatrimestre, para ser desarrollados con grupos de estudiantes diversos, tanto de nivel educativo como de edad.

La muestra, seleccionada por conveniencia, estuvo conformada por 82 estudiantes en el primer cuatrimestre y 140 en el segundo, estudiantes, como se aprecia en el cuadro, de diversas asignaturas y titulaciones: 
Tabla 1

Descripción de los grupos

\begin{tabular}{|c|c|c|c|}
\hline Titulación & $\begin{array}{l}\text { Curso, } \\
\text { cuatrimestre }\end{array}$ & Título de la asignatura & $\begin{array}{l}\text { Número de } \\
\text { estudiantes }\end{array}$ \\
\hline $\begin{array}{l}\text { Grado en Educación } \\
\text { Social }\end{array}$ & $4^{\circ}, 1^{\circ}$ & $\begin{array}{l}\text { Cambios sociales y nuevas } \\
\text { relaciones de género }\end{array}$ & $\begin{array}{l}26 \\
(24 \text { Mujeres } / 2 \\
\text { Hombres) }\end{array}$ \\
\hline $\begin{array}{l}\text { Programa } \\
\text { Universitario de } \\
\text { Mayores (Sede Jaén) }\end{array}$ & Optativa, $1^{\circ}$ & $\begin{array}{l}\text { Identidades } \\
\text { intergeneracionales } \\
\text { aprendiendo y enseñando } \\
\text { patrimonio cultural }\end{array}$ & $\begin{array}{l}56 \\
\text { (40 Mujeres } / 16 \\
\text { Hombres) }\end{array}$ \\
\hline $\begin{array}{l}\text { Grado en Educación } \\
\text { Primaria }\end{array}$ & $2^{\circ}, 1^{\circ}$ & $\begin{array}{l}\text { Didáctica de las ciencias } \\
\text { sociales I: el espacio geográfico } \\
\text { y su tratamiento didáctico }\end{array}$ & $\begin{array}{l}54 \\
(25 \text { Mujeres/29 } \\
\text { Hombres) }\end{array}$ \\
\hline $\begin{array}{l}\text { Grado Educación } \\
\text { Social }\end{array}$ & $3^{\circ}, 2^{\circ}$ & $\begin{array}{l}\text { Educación natural, cultural y } \\
\text { patrimonial }\end{array}$ & $\begin{array}{l}45 \\
(27 \text { Mujeres } / 18 \\
\text { Hombres) }\end{array}$ \\
\hline $\begin{array}{l}\text { Máster Oficial } \\
\text { Gerontología: } \\
\text { Longevidad, Salud y } \\
\text { Calidad }\end{array}$ & Optativa, $2^{\circ}$ & $\begin{array}{l}\text { Intervención Socio-pedagógica } \\
\text { y Cultural en Gerontología }\end{array}$ & $\begin{array}{l}6 \\
\text { (4 Mujeres } / 2 \\
\text { Hombres) }\end{array}$ \\
\hline $\begin{array}{l}\text { Programa } \\
\text { Universitario de } \\
\text { Mayores (Sede } \\
\text { Andújar) }\end{array}$ & Optativa, $2^{\text {a }}$ & $\begin{array}{l}\text { Identidades } \\
\text { intergeneracionales } \\
\text { aprendiendo y enseñando } \\
\text { patrimonio cultural. }\end{array}$ & $\begin{array}{l}35 \\
(24 \text { Mujeres / } \\
11 \text { Hombres) }\end{array}$ \\
\hline
\end{tabular}

\section{Fases de la investigación e instrumentos}

Dado que esperábamos conocer qué estereotipos y prejuicios aparecían en los distintos grupos y si con la implementación de dichas propuestas se producían cambios en los mismos, planificamos dos fases en la investigación.

A) Preparación de un itinerario por las localidades del alumnado del Programa de Mayores, de manera que ellos y ellas se convirtieran en guías por "su ciudad" para personas más jóvenes. En los grupos del Programa de Mayores, mediante un trabajo colaborativo vinculado a la investigación acción participativa debieron decidir qué patrimonio, qué valores y qué narraciones querían destacar. Optamos por el aprendizaje colaborativo (Barkley, Croos y Major, 2007) porque implica que la responsabilidad en el proceso de conceptualización es compartida, y es más importante el proceso que el resultado, ya que éste no deja de ser simbólico de un momento dado y no se asume como un producto final incuestionable. (Díez Bedmar, 2018a). Para ello eso se establecieron enfoques de trabajo por grupos que fueron: Paisaje, memoria y lugares; Oficios y trabajos; Mujeres y hombres; Contar la Historia y las historias. Los datos obtenidos se recogieron en un diario de observación sistemático en torno a tres ítems: Formación previa y capacidad de transmitir; Identificación de elementos patrimoniales y perspectiva de género.

B) Realización de los itinerarios intergeneracionales.

Para los grupos de Grado y Máster los itinerarios se presentaron como una práctica en la que analizar y observar los procesos de enseñanza-aprendizaje en una propuesta intergeneracional, para 
lo que se diseñó una guía de observación como instrumento de evaluación de los aprendizajes que desarrollarían y elaborar conclusiones, común en todos los grupos. El objetivo era también valorar si la titulación y curso influían en sus respuestas. Esta guía sería el instrumento de recogida de información en esta fase de la investigación.

Tabla 2

Items de observación

\begin{tabular}{|c|c|}
\hline $\begin{array}{c}\text { Ítem de } \\
\text { observación }\end{array}$ & Descripción \\
\hline $\begin{array}{l}\text { 1. Distribución de los } \\
\text { grupos }\end{array}$ & $\begin{array}{l}\text { ¿Cómo interaccionan los grupos de diferentes edades? ¿Cuándo? ¿En qué } \\
\text { sentido? ¿Quiénes dan el primer paso? }\end{array}$ \\
\hline $\begin{array}{l}\text { 2. Composición de } \\
\text { los grupos }\end{array}$ & $\begin{array}{l}\text { Observad el número de hombres y mujeres, cómo se agrupan en los } \\
\text { distintos colectivos, si las mujeres mayores se dirigen más a las chicas o a } \\
\text { los chicos, y al revés. ¿Por qué crees que pasa? }\end{array}$ \\
\hline \multirow[t]{2}{*}{ 3. Comportamiento } & $\begin{array}{l}\text { Observa y analiza los comportamientos que tienen ambos colectivos, en } \\
\text { cuestiones como: }\end{array}$ \\
\hline & $\begin{array}{l}\text { Uso del móvil; Atención a la práctica que se está realizando; Tiempo de } \\
\text { atención prestada; Participación; Interés por dar su punto de visto; Subidas } \\
\text { de voz; Risas y cotilleos }\end{array}$ \\
\hline $\begin{array}{l}\text { 4. En los centros de } \\
\text { interés }\end{array}$ & $\begin{array}{l}\text { Observa si se producen cambios mientras se transita de un lugar a otro con } \\
\text { respecto al momento en que nos paramos frente a un centro de interés para } \\
\text { trabajarlo }\end{array}$ \\
\hline $\begin{array}{l}\text { 5. Contenidos que } \\
\text { interesan más }\end{array}$ & $\begin{array}{l}\text { Observa, analiza y anota si los distintos colectivos tienen los mismos } \\
\text { intereses o si por el contrario a un colectivo le interesa más lo académico o } \\
\text { lo emocional }\end{array}$ \\
\hline 6. Vocabulario & $\begin{array}{l}\text { Analiza, observa y anota expresiones, giros lingüísticos, tipo de vocabulario } \\
\text { utilizado... Reflexiona sobre las semejanzas y diferencias }\end{array}$ \\
\hline $\begin{array}{l}\text { 7. Estereotipos y } \\
\text { prejuicios }\end{array}$ & $\begin{array}{l}¿ \text { ¿ué ideas previas se tienen a la hora de realizar una salida con mayores } \\
\text { adultos? Observa y analiza si se dan, y en qué medida, y si hay cambios al } \\
\text { principio y al final de tu presencia }\end{array}$ \\
\hline $\begin{array}{l}\text { 8.Temáticas de } \\
\text { observación }\end{array}$ & $\begin{array}{l}\text { Paisaje, memoria y lugares; Oficios y trabajos; Mujeres y hombres; Contar } \\
\text { la historia. Preparad preguntas que queráis realizar al colectivo al respecto } \\
\text { de cada una de estas temáticas. }\end{array}$ \\
\hline
\end{tabular}

Para finalizar se les solicitó que extrajeran conclusiones y presentaran una reflexión crítica final de la actividad con todo lo observado: ¿qué has aprendido para tener en cuenta si trabajas con mayores o si propones una actividad intergeneracional?

\section{Resultados}

\section{Resultados obtenidos en la Fase A}

En el contexto de esta actividad aparecieron las siguientes vinculaciones identitarias y prejuicios:

1.- Formación previa y capacidad de transmitir. Señalaron que solo quienes tenían una formación previa universitaria y/o habían ejercido una profesión (en su mayoría vinculada a la docencia en los 
niveles obligatorios) tenían el conocimiento académico suficiente para llevar a cabo las explicaciones.

2.- Identificación de elementos patrimoniales con monumentos. En un primer momento, para seleccionar qué patrimonio enseñar, apareció, principalmente, el concepto de monumento. 3.- La importancia de la perspectiva de género. Los roles de género presentes en sus vidas y en la manera de relacionarse con el patrimonio les vinculaba de manera diversa a lugares de la ciudad, así como a la memoria que tenían de ellos. Los lugares con los que nos identificamos o donde participamos como ciudadanos y ciudadanas, y "la identificación de olores y colores (a qué olía tal calle, a qué hora, quiénes estaban dónde en ese momento; quiénes encalaban las fachadas, las macetas en las ventanas) y los sonidos (tejer, coser, cortar, cantar, charlar entre la vecindad, dónde se sentaba cada quién, por qué...)" (Díez Bedmar, 2018b). Por ello el cómo se relacionaban a lo largo de sus historias de vida hombres y mujeres con los elementos patrimoniales, así como sus propias historias y las leyendas y narraciones, aparecieron también como elementos patrimoniales que antes no identificaban.

Como podemos apreciar, los estereotipos y prejuicios iniciales, fueron dando paso al reconocimiento de identidades patrimoniales diversas e interseccionales, que lejos de ser jerarquizadas, pasaron a ser complementarias, poniendo en juego la inteligencia emocional (Gardner, 1993) y, en el desarrollo de esa competencia emocional (Santamaría, 2010) comprendieron cómo el concepto construido de patrimonio con el que habían llegado al aula, heredero de su construcción escolar, tenía mucho de cultura patriarcal y heteronormativa.

\section{Resultados obtenidos en la Fase B}

Desde el punto de vista cuantitativo se ha utilizado un análisis de frecuencias tanto en la aparición de conceptos estructurantes del pensamiento que dan sentido a las respuestas y se ha porcentualizado el resultado en relación con el número total de informantes y su identificación de sexo-género.

Para el análisis de datos cualitativos, hemos utilizado MAXQDA 2012, en su versión de prueba, a fin de establecer etiquetas de indicadores de significación que nos ayuden a entender tanto el contexto como la terminología asociada a los conceptos. Además, la finalidad es realizar un Análisis Crítico del Discurso (Van Dijk, 1993), "fundamentalmente comprometido con analizar las relaciones de dominación, junto con las premisas de Baxter (2004, 2007), lo que ha llevado, primero, a establecer un corpus, anotando los conceptos junto a su construcción significativa derivada de la explicación y justificación de su respuesta y después a proceder al análisis de los resultados.

Para los ítems 1 y 2, el 95\% observó cómo "Las interacciones las comienzan las personas mayores y a partir de ahí comienzan las comunicaciones " (ItAnd, Inf3): Describen cómo "son los mayores quienes proceden a interactuar con nosotros/as, llamando nuestra atención para explicarnos el conocimiento que posee cada uno sobre el patrimonio de Andújar y expresando su preocupación por la adquisición de nuestro conocimiento" (ItAnd, Inf6), y el proceso "el grupo de edad más avanzada fue el que dio el primer paso y el grupo de menor edad empezó a interaccionar durante toda la visita didáctica de manera activa y prolongadamente" (ItAnd, Inf9).

Importante es constatar cómo "los hombres interaccionaban de manera más usual con los jóvenes chicos y las mujeres generalmente con las chicas” (ItJa, Inf67) afirmación que, de diversas maneras aparece en el 100\% de los casos, si bien en cierto que en algún caso se señala que "aunque nosotros estamos más mezclados, los mayores están separados por sexo, aunque en algún caso sean pareja" (ItJa Inf36) o que "Los jóvenes entre sí con personas de ambos sexos, en mayores con personas de su mismo sexo ya sean mayores o menores"(ItAnd, Inf8). El 100\% cree que es por cuestiones culturales y tradicionales aprendidas y señalan los cambios experimentados con respecto a la actualidad. 
En las respuestas de estas dos cuestiones no hay diferenciación ni en el grado académico ni en la expresión de género de quienes observan. Estos resultados son de suma importancia a la hora de programar una intervención intergeneracional para cualquier nivel educativo, y muestran la necesidad de reforzar acciones coeducativas en la Educación de Adultos.

Las respuestas a los ítems 3, 4 y 6 nos devuelven como resultado que la utilización del móvil se realizó para fotografiar ( $87 \%$ de las respuestas) si bien reconocen que el grupo de jóvenes lo usó para chatear. Una informante señala "no se realizó uso del móvil por parte de ninguno de los diferentes colectivos excepto para fotografiar algún elemento de interés o bien para mostrar información relacionada con la práctica en sí. Lo cual resultó muy satisfactorio” (ItJa, Inf 23).

El 100\% reconocen "un mayor grado de atención prestada del colectivo de la generación mayor" (ItJa, Inf 28), destacándose "el interés y las ganas de nuestros mayores por mostrarnos cada conocimiento que poseían al respecto" (ItAnd, Inf21) y una mayor participación con este mismo grupo "la mayor parte de la participación es del grupo de los mayores" (ItJa, Inf 5).

También aparece en el 100\% de los casos el altísimo grado de interés del colectivo mayor por trasmitir sus puntos de vista y sus experiencias: "Generalmente, mostraban muchísimo interés en transmitir sus puntos de vista, sus experiencias o vivencias relacionadas con lo que se iba viendo durante la guía didáctica. Y justo eso es lo verdaderamente enriquecedor" (ItJa, Inf 17); "los mayores ya que sabían que los jóvenes no éramos de allí y nos acogieron y explicaron gustosamente, ya que se sentían importantes y con protagonismo por ello" (ItAnd, Inf 11). El 5\% manifiesta además una diferencia en este interés por transmitir, al señalar que era "los mayores por experiencia personal en muchas ocasiones y los jóvenes por opinión personal” (ItJa, Inf 3).

En cuanto al comportamiento, indican que no hubo subidas de tono y que había "cotilleos al principio separados, luego conjuntamente. Al finalizar la salida didáctica, todo el grupo (mayores y jóvenes) comenzó a reírse y a cantar una canción que nos enseñaron" (ItAnd, Inf 11), además, "los jóvenes utilizaban las diversas jergas pertenecientes a la edad, mientras que las personas mayores adaptaban su vocabulario a las distintas situaciones que aparecían" (ItAnd, Inf 17).

Las respuestas al ítem 6, "Vocabulario" lleva en el 100\% de los casos a reconocer que "Los mayores manejan más vocabulario que las personas jóvenes debido a la edad que tienen estos han aprendido más vocabulario y conocen mejor el cambio que han sufrido las palabras" (ItJa, 63), si bien aparecen también la sorpresa "me sorprendió que conocieran términos vinculados a la tecnología del móvil” (ItJa, Inf 39) y los prejuicios "Es cierto, que el grupo de alumnos jóvenes pensamos de ante mano que las personas mayores usarían palabras que no entenderíamos, y viceversa." (ItAnd, Inf 21).

Como conclusión, "los mayores tenían tanto que contarnos y estaban tan entusiasmados que se acercaban a los jóvenes más cercanos para enseñarles algo de lo que querían hablarle” (ItJa, Inf 7), "quizás durante estos momentos de tránsito de un lugar a otro, se establecía la mayor comunicación entre los participantes. Las conversaciones surgían de forma natural y se interactuaba con cada persona que encontrabas al lado, independientemente de la edad o sexo. Durante estos momentos, esa interacción era más reducida en cuanto al número de participantes, pero quizás bastante más constructiva, ya que permite profundizar más sobre la persona en sí y se establece un mayor grado de confianza." (ItAnd, Inf18). Es por eso por lo que consideremos que este tipo de propuestas educativas dan protagonismo a los colectivos de mayores al mismo tiempo que facilitan la reflexión sobre los aprendizajes y el conocimiento de la legua, la historia, la cultura, las tradiciones en los colectivos de menores y jóvenes. Esta autoevaluación facilita, además, la vinculación con la competencia de aprendizaje continuo a lo largo de la vida, y el desarrollo de la empatía en la narración y la memoria histórica. 
Las respuestas al ítem 5, "Contenidos que interesan más" nos llevan a los centros de interés por parte de cada colectivo y a una diferenciación observable no solo con respecto a las edades, sino también con respecto al sexo-género.

Desde un punto de vista cuantitativo, los resultados obtenidos en estas observaciones, nos indican que los y las mayores muestran más interés y transmiten más elementos de Patrimonio cultural sobre todo usos sociales, tradiciones, actos festivos, técnicas artesanales y lo hacen con una fuerte vinculación emocional e identitaria, mientras que el colectivo más joven, está más interesado en el "valor académico" y lo relacionan más con el Patrimonio Histórico-Artístico y Arqueológico: "Desde mi punto de vista, al colectivo de estudiantes le interesa más lo puramente académico y al colectivo de personas mayores, lo emocional puesto que para ellos el valor cultural e histórico es más emocional que académico" (ItJa, Inf 25); "Por parte del alumnado de la universidad de adultos, podemos observar como su interés es más emocional que la otra parte del alumnado, pudiendo ser en ellos algo más académico, pero conforme avanza la visita, se van contagiando de este lado emocional que transmiten los mayores" (ItAnd, Inf 27).

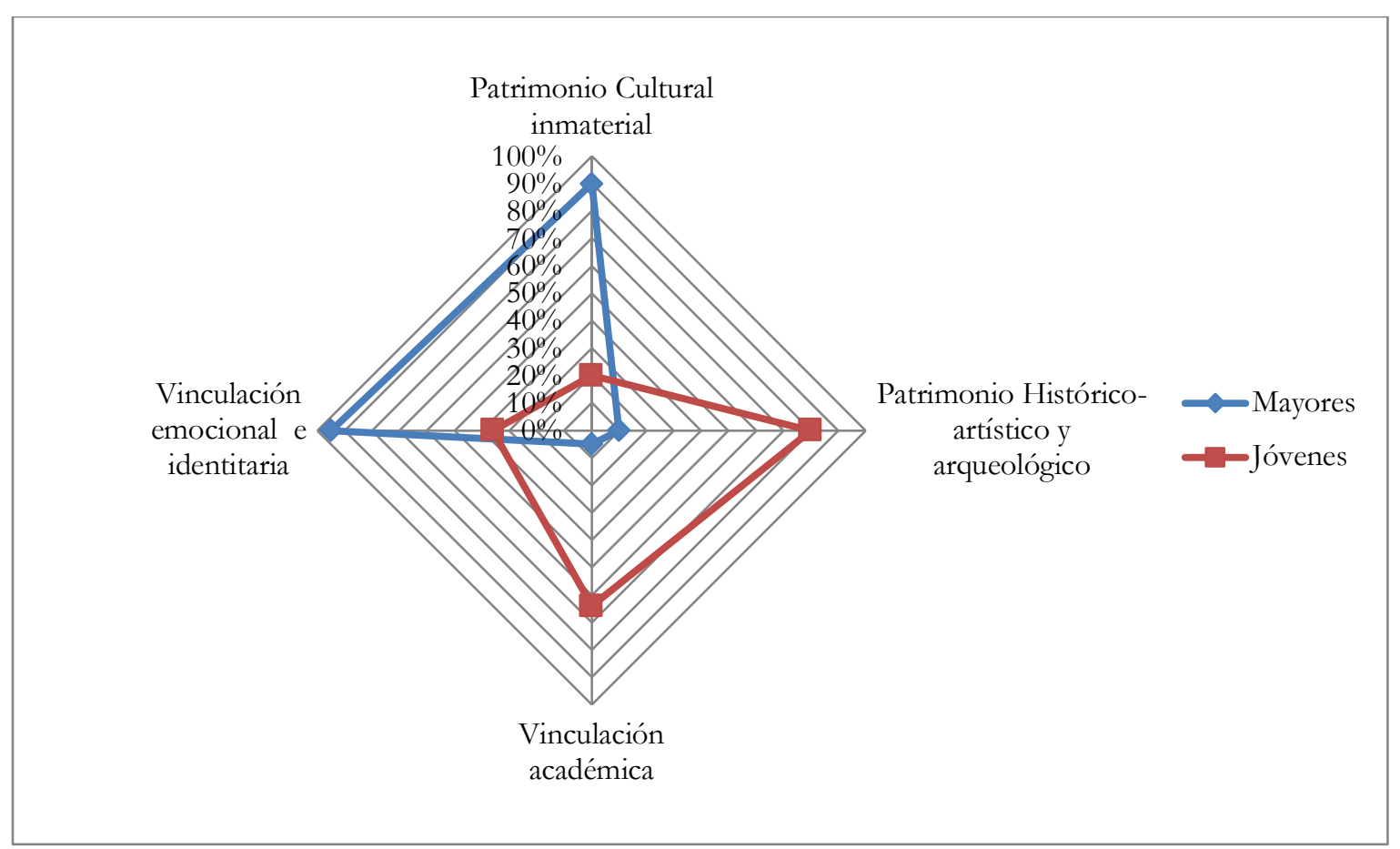

Gráfica 1. Resultados obtenidos en el ítem 5

Aparece también la comprensión de la diferenciación sexo-género en las relaciones con el patrimonio "Los centros de interés eran diferentes, aunque mostrando ambos la misma motivación, las personas de cada sexo montaban su discurso en base a su experiencia personal. Por lo cual, podríamos decir que dependiendo del papel que haya tenido cada persona a lo largo de su vida, encontramos gustos y preferencias variados." (ItAnd, Inf 14). "Ellas nos contaron la piscina donde podían bañarse, que no era mezclada con los chicos, y la sección femenina que era como una mili de mujeres, ellos hablaron de dónde estaban las prostitutas" (It Ja, Inf 12).

Por último, las respuestas a los ítems 7 y 8 también nos muestras diferencias, y en esta ocasión, además, podemos observar la diferenciación entre el curso de la misma titulación y el nivel de grado o postgrado cursado. Basta con observar las frecuencias de aparición de las palabras. 


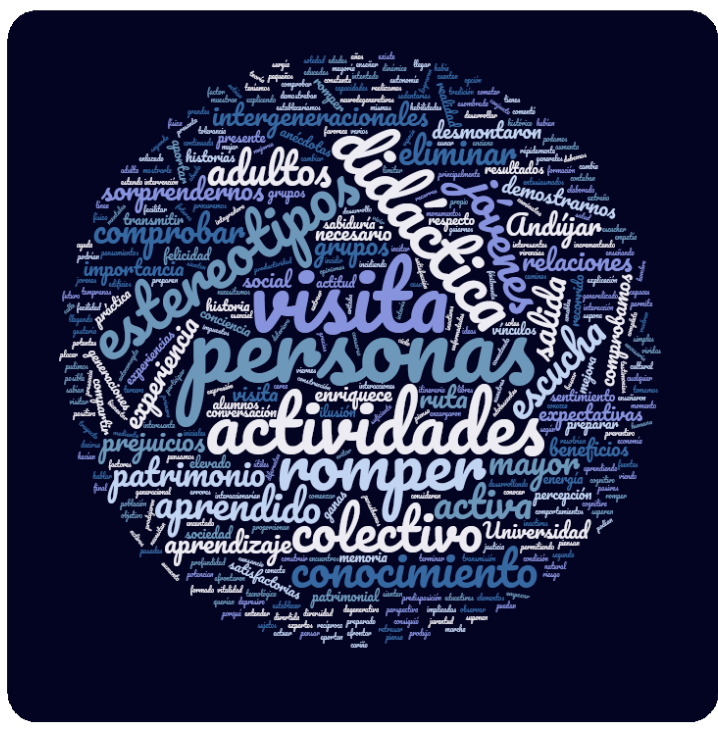

Gráfico 2. Nube de palabras de Educación natural, cultural y patrimonial, $3^{\circ}$ Educación Social, $2^{\circ}$ cuatrimestre

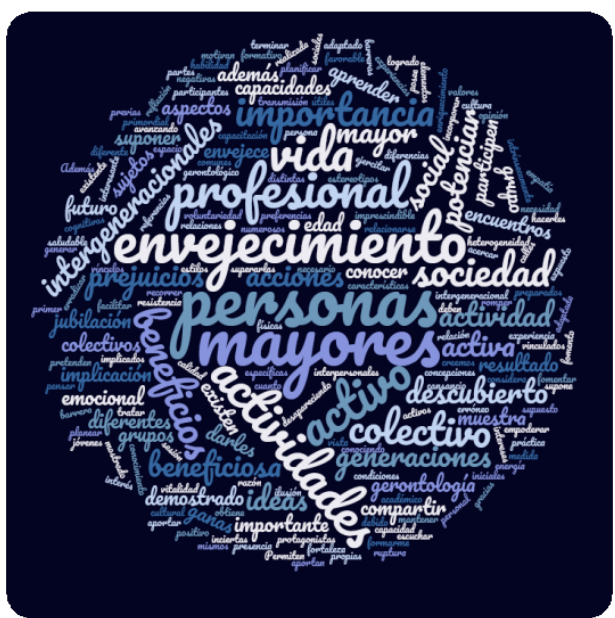

Gráfico 4. Nube de palabras de Intervención Socio-pedagógica y Cultural en Gerontología. Máster: Gerontología: Longevidad, Salud y Calidad

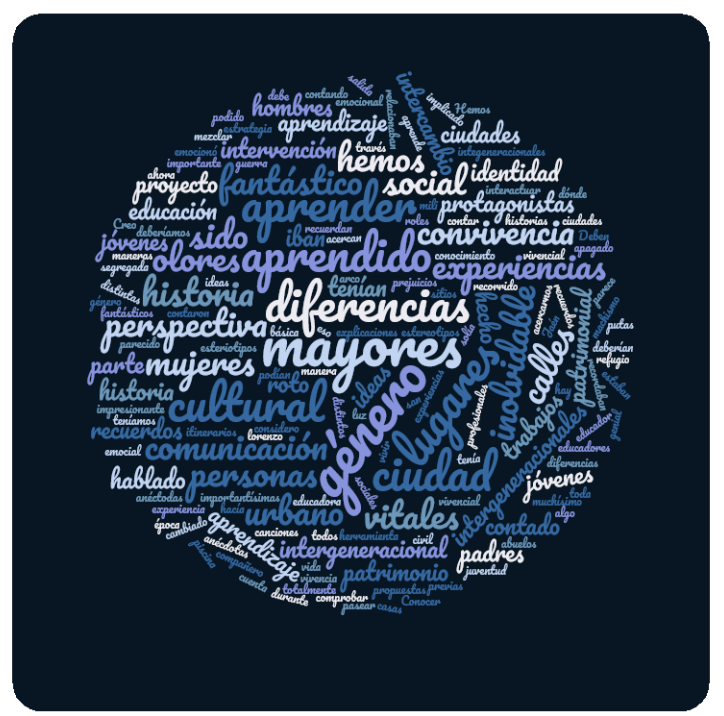

Gráfico 3. Nube de palavras de Cambios sociales y nuevas relaciones de género. $4^{\mathrm{o}}$ Educación Social. 1er cuatrimestre

Podemos comprobar cómo las respuestas aparecen matizadas en función de la asignatura que se está cursando, puesto que se relacionan con todo lo trabajado en la materia, en el caso de las dos primeras imágenes, y con el futuro profesional, en el caso del Máster. Así mismo, el vocabulario utilizado en las respuestas muestra un mayor grado de especialización en el Máster. 


\section{Discusión}

Para el alumnado del Programa de Mayores, ante la pregunta final de qué es lo que han aprendido de la experiencia y lo que quieren transmitir a las y los jóvenes (Puig, 2007), la totalidad de las respuestas aparecen vinculadas a una reflexión sobre las injusticias y las discriminaciones con respecto a los aprendizajes sobre patrimonio de las que no habían sido conscientes y señalan la importancia de transmitir las relaciones de vida con el patrimonio para que no se pierdan, sintiéndose protagonistas y agentes activos en este sentido. Aparecen, por tanto, el valor de la Memoria y la transmisión oral con respecto a la Educación Patrimonial.

En los grupos de grado y máster manifiestan, de una u otra manera, que han sido conscientes de sus prejuicios y de la importancia que éstos tenían en lo que esperaban de esta propuesta: "Todos teníamos la idea de que al ser personas mayores haríamos una ruta más corta y tranquila, puesto que consideramos que suelen cansarse rápidamente. Nos llevamos una gran sorpresa ya que hubo ocasiones en las que mostraron menor dificultad de movimiento que nosotros." (ItAnd, Inf 4); "Gracias a esta visita, los alumnos de la universidad de Jaén comprobamos que no hay que tener prejuicios y estereotipos sobre estas personas, puesto que cuando pensamos en llevar a cabo una intervención o un itinerario con el colectivo de la tercera edad, procuramos realizar actividades tranquilas para ellos. Pero este grupo de mayores consiguió romper todos los esquemas que llevábamos y nos enseñaron que nunca hay que limitarlos, ya que son capaces de realizar las mismas actividades que nosotros, incluso con mayor facilidad que la población joven" (ItAnd, Inf 22). "Para terminar, destaré algunos aspectos y conclusiones finales sobre esta actividad intergeneracional que en mi opinión ha resultado beneficiosa para ambos grupos aunque sinceramente considero que el mayor beneficiado he sido yo con la ruptura de esos prejuicios y estereotipos iniciales sobre el colectivo de personas mayores" (ItAnd, Inf5).

El 100\% indican que han entendido la importancia para elaborar proyectos de educación patrimonial intergeneracionales "enriquecimiento cultural de los diferentes colectivos, entender que el patrimonio somos todos y todas y no solo de las administraciones y que conocer los distintos puntos de vista e intentar comprenderlos, acabar con los estereotipos que se tienen de otros grupos, indagar a cerca de las formas de vida y tradiciones ajenas, entre otros.” (ItJa, Inf 18).

Los aprendizajes patrimoniales han adquirido nuevas dimensiones para ellos y ellas: "El patrimonio ahora tiene para mí más sentido, no es solo el monumento que se ve, en la calle donde está, y cómo ese edificio tiene significados de vida e historias" (ItJa, Inf 17); "He aprendido muchísimo de patrimonio, más que en toda me vida, y no ha sido por obligación, solo lo he aprendido disfrutando" (ItAnd, 31); "He comprobado lo poco que sabía de Jaén, y mira que soy de aquí, solo sabía los monumentos pero no sabía la riqueza patrimonial que encerraba cada lugar. Nunca volverá a ser lo mismo". (ItJa, Inf1); "Yo creía que el patrimonio era lo mismo para todos, no entendía cómo podía ser tan enriquecedor entender las diferentes miradas a lo mismo por edad y por sexo, lo que para unos es el casco histórico, para otros es toda su historia de vida" (ItJa, Inf 15). "He podido comprobar la importancia de la perspectiva de género al hablar de patrimonio, porque cuando hombres y mujeres hablan de patrimonio, aunque sea con respecto a la misma ciudad o lugar, narran cosas distintas. Por tanto, si lo explico, es que estarán vinculándolo a recuerdos distintos y los aprendizajes serán diferentes" (ItJa, Inf 43). 


\section{Conclusiones}

$¿$ ¿Acaso existen en el universo realidades no-complejas? (...) la respuesta contundente es negativa. Dicho de otro modo, si cualquier realidad universal se tuviese como simple, sería porque se estuviese juzgando parcialmente, desde un relativo desconocimiento o con una intencionalidad esquematizadora (De la Herran Gáscon, 1999, p. 39)

En la formación inicial del profesorado, la mayoría de las veces, formamos para que el alumnado ejerza su función con grupos de edad cerrados. En este sentido, por ejemplo, en el Grado en Educación Primaria, pensamos en menores de entre 6-12 años, pero olvidamos que también podrán ejercer su profesión en Educación de Adultos. Así mismo, cuando formamos en el Máster de Formación de Profesorado de e Educación Secundaria Obligatoria y Bachillerato, Formación Profesional y Enseñanza de Idiomas, también olvidamos que existen la Educación Secundaria para Personas Adultas (ESPA) y Bachillerato (BTOPA), y que, en Formación Profecional, gran parte del alumnado es adulto mayor (Milana \& Holford, 2014). Además, las actividades intergeneracionales están cada vez más presentes en las aulas (Bholinger, Haake, Helms Jørgensen, Toiviainen \& Wallo, 2015; Sánchez, Whitehouse \& Johnston, 2018), ya sea en Comunidades de Aprendizaje, como en la implicación de las familias en proyectos de Centro y el aprendizaje intergeneracional es reconocido como una herramienta para construir y transformar el patrimonio cultural (Labrador, Silberman, Cortellesi, Harpley \& Kerman, 2018).

Por otra parte, cuando trabajamos desde un punto de vista teórico conceptos como Educación Patrimonial, intergeneracionalidad o interseccionalidad, olvidamos que, como evidenció Sola (2005, p. 200), citando a Porlán "la idea del aprendizaje como algo lineal e irreversible impide comprender los procesos, a veces sinuosos, contradictorios y reversibles, que se manifiestan en la evolución de cada alumno en particular".

La Didáctica de las Ciencias Sociales tiene un papel protagonista en la formación inicial de aquellas personas que van a trabajar Educación Patrimonial, ya sea en contextos formales o informales de aprendizaje, y es en las materias del área donde el alumnado asume el "rol de mediador o mediadora en el conocimiento y adquisición de identidades complejas, (...) se construye, de manera crítica, y se toma de conciencia de la diversidad cultural e identitaria tanto en el espacio, como en el tiempo histórico, además de adquirirse la consciencia de los cambios que se producen en el tiempo" (Díez Bedmar, 2016).

Con propuestas como la analizada se hace patente que, como señalaba Warren (2000, pp. 99100) "Las relaciones con las/os demás son el eje desde donde cada cual se conoce a sí mismo". Desarrollar propuestas educativas de educación patrimonial intergeneracionales ayuda al alumnado a analizar la realidad bajo el prisma de la interseccionalidad, le ayuda a autoevaluarse en sus conocimientos previos, a pensar en el grupo al que dirigirá sus propuestas y a entender los centros de interés diferenciados. Comprenderá que en la planificación no se debe tener en cuenta solamente qué enseñar, sino las dificultades de aprendizaje que pueden aparecer en cada persona, que enlaza los nuevos aprendizajes con sus conocimientos previos y sus historias de vida. Todo ello supone una autoevaluación personal y profesional sobre qué contenidos y porqué se van a priorizar y cuáles son los objetivos que se buscan alcanzar. Los grupos-clase dejan así de ser percibidos como homogéneos, y aparecen las identidades, los significados construidos y las premisas a tener en cuenta: edades, contextos culturales y sociales, sexo-género, conocimientos previos... como requisito indispensable para entender que el patrimonio no tiene una única manera de aprenderse, comprenderse, vivirse y trasmitirse. Todo ello implica la asunción de responsabilidades y el desarrollo del pensamiento crítico. 
Por último, la toma de conciencia de estereotipos, prejuicios (por edad, por sexo-género, por nivel de estudios o profesión) y la importancia de los conocimientos previos (cuáles creemos que son más importantes y cuáles destacamos), facilita la autoevaluación, el aprendizaje permanente y los valores educativos que tiene el patrimonio.

\section{Referencias}

Barkley, E., Croos, P. y Major, C. (2007). Técnicas de aprendizaje colaborativo. Madrid: Morata. Baxter, J. (2004). Positioning Gender in Discourse: A Feminist Methodology. Londres: Palgrave Macmillan. Baxter, J. (2007). Post-structuralist analysis of classroom discourse. In M. Martin \& A.M. Mejia (Eds.), Encyclopaedia of Language and Education: Discourse and Education, (3), pp. 69-80. Nueva York: Springer

Bedmar, M. (2003). La Educación Intergeneracional encierra un tesoro. En M. Bedmar, y I. Montero (Coords.), La Educación Intergeneracional: un nuevo ámbito educativo (pp. 67-82). Madrid: Dykinson.

Bedmar, M. (2010). La Educación Intergeneracional. En M ${ }^{a}$ C. Fernández y J. Ga Mínguez (Comps.), Educación y Adultos Mayores (pp. 169-205). Rosario: Laborde Editor.

Bholinger, S., Haake, U., Helms Jørgensen, C., Toiviainen, H.\& Wallo, A. (Eds.) (2015). Working and Learning in Times of Uncertainty. Challenges to Adult, Professional and Vocational Education. Rotterdam: Sense Publisher.

Crenshaw, K. (1989). Demarginalizing the Intersection of Race and Sex: A Black Feminist Critique of Antidiscrimination Doctrine, Feminist Theory and Antiracist Politics. University of Chicago Legal Forum, 1(8), 139-167.

De la Herran Garcón, A. (1999). Claves para la formación total de los profesores. Tendencias pedagógicas, 4, 37-58.

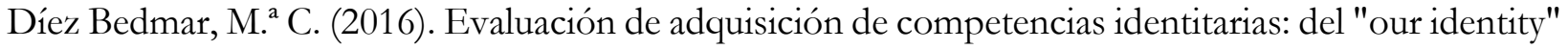
al "identities". En C. García, A. Arroyo y B. Andreu (Eds.), Deconstruir la alteridad desde la Didáctica de las Ciencias Sociales: educarpara una ciudadania global (pp. 320-330). Las Palmas de Gran Canaria: AUPDCS y Universidad de Las Palmas de Gran Canaria.

Díez Bedmar, M. ${ }^{a}$ C. (2018a). Del concepto construido de familia a la construcción colaborativa: análisis y recursos desde una educación emocional, feminista y sociocrítica. En S. Medina Quintana (Ed.), Familias, educación y género. Tradiciones y rupturas en las sociedades moderna y contemporánea (pp. 151-172). Gijón: Trea.

Díez Bedmar, M. ${ }^{a}$ C. (2018b). Paisajes culturales urbanos con perspectiva de género: Revisión bibliográfica y repercusiones para la didáctica de las ciencias sociales. UNES, 4, 60-77.

Díez Bedmar, M. ${ }^{a}$ C. (2019). Educación histórica con perspectiva de género: Resultados de aprendizaje y competencia docente. El Futuro del Pasado, 10, 81-122. http://dx.doi.org/10.14516/ fdp.2019.010.001.003

Estepa Giménez, J. (2017). Otra didáctica de la Historia para otra escuela. Lección inaugural. Servicio de Publicaciones Universidad de Huelva.

Fontal, O. (2013). Estirando hasta dar la Vuelta al Concepto de Patrimonio. En O. Fontal (Coord.), La Educación Patrimonial: Del Patrimonio a las Personas (pp. 9-22). Gijón: Trea.

Fontal, O. e Ibáñez, A. (2015). Estrategias e instrumentos para la educación patrimonial en España. Educatio Siglo XXI, 33(1), 15-32.

Gardner, H. (1993). Inteligencias múltiples. La teoría en la práctica. Madrid: Paidós Educación.

Guerra, M. J. (2016). Identidades, educación y enfoques interseccionales: del diagnóstico de las opresiones a la vindicación de los derechos. En C. García, A. Arroyo y B. Andreu (Eds.), Deconstruir la alteridad desde la Didáctica de las Ciencias Sociales: educar para una ciudadanía global (pp. 
203-218). Las Palmas de Gran Canaria: AUPDCS y Universidad de Las Palmas de Gran Canaria.

Labrador, A., Silberman, N., Cortellesi, G., Harpley, J. \& Kernan, M. (2018). Intergenerational Learning: A Tool for Building and Transforming Cultural Heritage. En A. Labrador \& N. Silberman (Ed)., Handbook of Public Heritage Theory and Practice. London: Oxford University Press.

Lucas, L. (2018). La enseñanz̧a del patrimonio y de la ciudadanía en las clases de ciencias sociales: un estudio de caso en ESO. Tesis doctoral, Universidad de Huelva.

Martín, A.V. (1999). Más allá de Piaget: cognición adulta y educación. Teoría de la Educación, 11, 127157.

Martín, M. y Cuenca, J. M. (2015). Educomunicación del patrimonio. Educatio siglo XXI, Actualidady tendencias en educación patrimonial, 33(1), 33-53.

Martins, T., Midão, L., Martínez, S., Dequech, L., Busse, G., Bertram, M., McDonald, A., Gilliland, G., Orte, C., Vives, M., \& Costa, E. (2019). Intergenerational Programs Review: Study Design and Characteristics of Intervention, Outcomes, and Effectiveness. Journal of Intergenerational Relationships, 17(1), 93-109.

Milana, M. \& Holford, J. (Ed) (2014). Adult Education, Policy and the European Union: Theoretical and Methodological Perspectives, Rotterdam: Sense Publisher.

Ortega y Gasset, J. (1923). El tema de nuestro tiempo. En Obras Completas, tomo III, 559-652 (2005). Madrid: Santillana.

Puig, J. M. (2007). Aprendizaje servicio: educar para la ciudadanía. Barcelona: Octaedro.

Sánchez M., Whitehouse, P. \& Johnston, L. (2018). Intergenerational Learning and Education in Schools and Beyond. Journal of Intergenerational Relationships, 16, 1-2.

Santamaría, J. S. (2010). La competencia emocional en la escuela: una propuesta de organización dimensional y criterial. Ensayos: Revista de la Facultad de Educación de Albacete, 25, 79-96.

Santisteban, A., González-Monfort, N. \& Pagès, J. (2019). Critical citizenship education and heritage education. En E. J. Delgado-Algarra \& J. M. Cuenca-López (Eds.), Handbook of Research on Citizenship and Heritage Education (pp. 26-42). Hershey PA, USA: IGI Global. 10.4018/978-17998-1978-3.ch002

Sola, C. (2006). Fundamentos de la técnica didáctica ABP. En C. Sola (Coord.), Aprendiraje basado en problemas (pp. 38-50). México: Trillas.

UNESCO (2016). Informe mundial sobre la cultura para el desarrollo urbano sostenible. París: Organización de las Naciones Unidas para la Educación, la Ciencia y la Cultura. Disponible en: https://unesdoc.unesco.org/ark:/48223/pf0000248920

Van Dijk, T. A. (1993). Principles of Critical Discourse Analysis. Discours \& Society, 4(2), 249-283.

Warren, K. (2000). Ecofeminist philosophy: a wester perspective on what it is and why it matter. Lanham, Md: Rowman and Littlefield. 


\section{Información sobre la autora}

Autora: María del Consuelo Díez Bedmar

Institución: Área de Didáctica de las Ciencias Sociales. Universidad de Jaén Email: modiez@ujaen.es

ORCID: https://orcid.org/0000-0002-5599-7624 


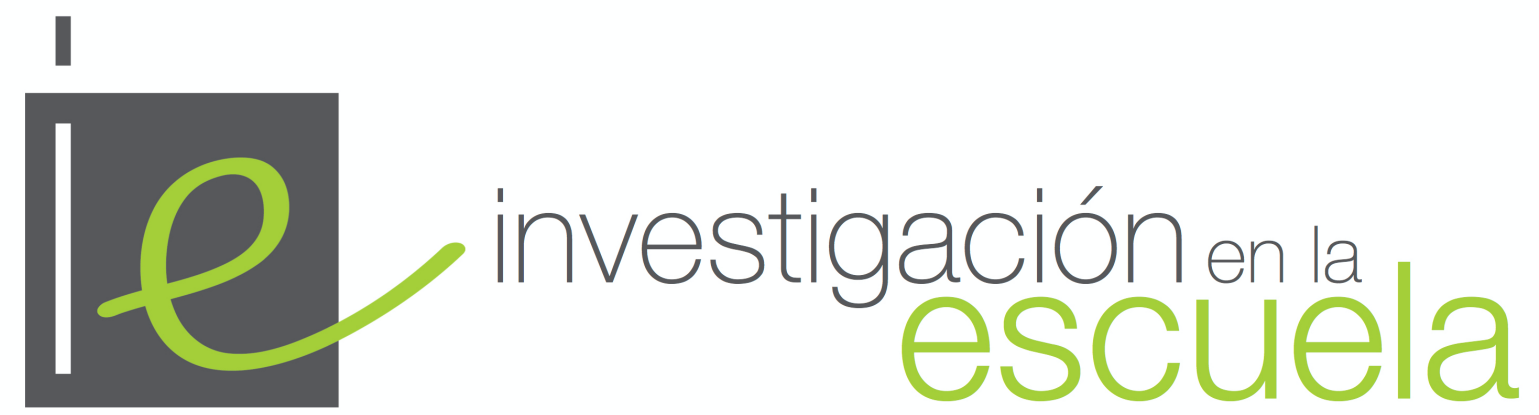

Revista académica evaluada por pares y de acceso abierto

Número 100

30 de abril de 2020

ISSN 2443-9991

\section{cc) (i) (2)}

Esta obra está bajo una licencia Creative Commons. Los/as lectores/as pueden compartir, copiar y redistribuir el material en cualquier medio o formato, así como adaptar, remezclar, transformar y construir a partir del material para cualquier propósito, incluso comercialmente. Para ello, deben de hacerlo bajo los siguientes términos: dando crédito de forma adecuada, brindando un enlace a la licencia e indicando si se han realizado cambios. Si se remezcla, transforma o crea a partir del material, debe distribuir su contribución bajo la misma licencia del original.

Más detalles de la licencia de CreativeCommons se encuentran en https://creativecommons.org/licenses/by-sa/4.0/deed.es

Cualquier otro uso debe ser aprobado en conjunto por el autor/es, o Investigación en la Escuela.

Uิ?

Revista Editada por la Universidad de Sevilla. https://editorial.us.es/es/revistainvestigacion-en-la-escuela

Por errores y sugerencias contacte a investigacionenlaescuela@,us.es

La revista Investigación en la Escuela desde su origen en 1987 hasta su nº 87 (2015) fue editada por Díada Editora. 
